\title{
Cognitive bias modification training in adolescents reduces anxiety to a psychological challenge.
}

\author{
Jennifer Y. F. Lau, Stefano R. Belli and Rajesh Chopra \\ Department of Experimental Psychology, University of Oxford, Oxford, UK
}

\begin{abstract}
Background: Anxiety disorders commonly occur in adolescence with disabling consequences. As adolescent anxiety predicts adult anxiety and mood disorders, there are growing efforts to identify suitable tools for prevention. Prior data show the effectiveness of Cognitive Bias Modification of Interpretations (CBM-I) training in generating positive interpretative styles of ambiguous information in adolescents. Here, we assess the effects of positive CBM-I training on anxious mood responses to a psychological challenge performed under time constraints and anticipated social evaluation.

Methods: Fifty adolescents were randomly assigned to receive positive or negative computerised cognitive training. Training involved reading and listening to 60 ambiguous scenarios that were resolved via completion of a word fragment in a positive or negative direction. After training, all adolescents completed an interpretation bias test and performed a difficult mental arithmetic task while believing that they were being videotaped for teaching purposes. Before and after training, and before and after the psychological challenge, adolescents completed anxiety ratings using visual analogue scales.

Results: Data from the interpretation bias test showed that training was successful at inducing positive and negative interpretations in each training group respectively. There were no effects of training group on changes in anxious mood before and after training. However anxious mood changes assayed before and after the psychological challenge revealed training effects: compared to negatively-trained adolescents, positively-trained adolescents showed attenuated anxiety levels post but not pre-stressor.

Conclusion: These results confirmed previous data that computerised CBM-I training can generate positive and negative interpretative styles in adolescents. These data also showed that induced interpretations could impact on anxious responses precipitated by a psychological challenge. These data have implications for using CBM-I training as a preventative tool targeting emotional well-being across all adolescents.
\end{abstract}

Key words: cognitive bias modification training, interpretative style, anxiety, adolescence, prevention, stress

Abbreviations: CBM-I: Cognitive bias modification of interpretations 


\section{Introduction}

Anxiety problems are remarkably common in youth (Merikangas, Nakamura, \& Kessler, 2009). Anxiety is characterised by intense and persistent fears and worries, and if untreated, can lead to peer difficulties (Wood, 2006), poor school attendance and performance (Van Ameringen, Mancini, \& Farvolden, 2003), other psychological problems such as alcohol or drug use (Zimmermann et al., 2003) and adult anxiety and mood disorders (Pine, Cohen, Gurley, Brook, \& Ma, 1998). Adolescent anxiety therefore generates considerable costs for the economy (Greenberg et al., 1999), prompting calls for early treatment and prevention (Sahakian, Malloch, \& Kennard, 2010). Recent excitement has been generated by the therapeutic potential of computerised cognitive training tasks (Bar-Haim, 2010). Such tasks alter information-processing styles typically linked to anxiety, for example, by training individuals to direct attention away from (rather than towards) threats or by selecting positive (rather than negative) interpretations of ambiguous material. Amassing data from youth samples attests to the effectiveness of changing attention-orienting biases to threat on anxiety reduction (Bar-Haim, 2010), but fewer studies have explored the benefits of training tasks that target biases in interpretation. In the present study, we assess the capacity of an audiovisual computerised Cognitive Bias Modification of Interpretations (CBM-I) training task in reducing anxious responses to a laboratory stressor in adolescents.

CBM-I training, pioneered in adults, presents participants with a series of ambiguous stimuli (words or scenarios) that comprise the training items (Grey \& Mathews, 2009; Mathews \& Mackintosh, 2000). During training, stimuli are resolved either in a positive or a negative direction, most commonly by completion of a word fragment at the end of the scenario (Mathews \& Mackintosh, 2000). Word completion disambiguates the meaning of the described situation. This is followed by a comprehension question, which can only be answered correctly if the ambiguous situation had been interpreted in the intended direction, thus reinforcing the direction of resolution. Adult data have demonstrated the effectiveness of CBM-I training on follow-up tests of interpretation style. On these, positively-trained participants endorse more positive than negative interpretations of new ambiguous situations while negatively-trained participants endorse more negative than positive interpretations. This plasticity of interpretative style has been reported in individuals with varying anxiety levels (MacLeod, Koster, \& Fox, 2009). These data also show that induced interpretative styles generate differences in anxious mood after training (Mathews \& Mackintosh, 2000; Mathews, Ridgeway, Cook, \& Yiend, 2007; Salemink, van den Hout, \& Kindt, 2007; Standage, Ashwin, \& Fox, 2009) and in response to symptom provocation (Hirsch, Hayes, \& Mathews, 2009; Murphy, Hirsch, Mathews, Smith, \& Clark, 2007; Wilson, MacLeod, Mathews, \& Rutherford, 2006). Thus compared to negatively-trained participants, positively-trained participants show reductions in anxious responses to naturally-occurring stressors or psychological challenges.

Drawing on work in adults, we have begun to investigate training efficacy in adolescents, given the current foci of preventative work in this age range. While there have been other reports of successful modification of interpretation biases in youth (Muris, Huijding, Mayer, \& Hameetman, 2008; Muris, Huijding, Mayer, Remmerswaal, \& Vreden, 2009; Vassilopoulos, Banerjee, \& Prantzalou, 2009), these have used different training paradigms to those used in adults and have focused on prepubescent children. We have therefore adapted the adult training task for use in adolescents by the inclusion of age-appropriate training material (see Figure 1). Across three studies of over 160 adolescents aged 13-18 years, we have found robust training effects on subsequent interpretative style (Lau, Molyneaux, Telman, \& Belli, 2010; Lothmann, Holmes, Chan, \& Lau, 2011; Telman, Holmes, \& Lau, 2011). However, mood effects associated with training have been less clear, with one study reporting reduced negative affect after positive training (Lothmann et al., 2011) and the other two finding reporting decreased positive affect (Lau et al., 2010) and increased negative affect (Telman et al., 2011) in negatively-trained adolescents only. In this third study, training did however impact responses to recent, naturally-occurring stressors. These preliminary data showed that adolescents who had received positive training rated recent life events as less disabling than negatively-trained adolescents. If replicated, these results suggest that training effects on anxious mood may be best measured during symptom provocation. These data are limited however by the possibility that adolescents assigned to the negative training group also experienced more severe stressors, although 
no differences in the overall number of life events were found across groups in these data.

The current study followed up these initial findings by investigating whether CBM-I training could alter anxious responses to a laboratory-induced stressor. As previous studies have found that mental arithmetic tasks performed to an audience reliably elicits mild stress (Stroud et al., 2009), these procedures were adopted. We also developed a new version of our previous training task that included supplementary auditory sound clips for each scenario. While our previous training task yielded promising changes in interpretative style, its reliance on reading ability may limit its use amongst adolescents with reading difficulties, who are younger or who do not speak English as a first language. While adult data suggest that there are no advantages associated with auditory training (Standage et al., 2009), theories of multimedia learning suggest more effective processing of information in bimodal rather than unimodal stimuli presentation (Mayer, 2008). The present study therefore investigated the effectiveness of positive audiovisual CBM-I training on subsequent interpretative style and on attenuating anxious responses during a psychological challenge. We used an unselected sample of adolescents from the community to better inform strategies that improve social and emotional wellbeing across all adolescents rather than only those with diagnosed problems. A negative training condition comprised the 'control' condition. As non-clinical individuals are often found to exhibit a 'positivity bias' (Mezulis, Abramson, Hyde, \& Hankin, 2004), use of a neutral or benign training condition may reduce detection of training effects associated with positive training.

\section{Methods}

\section{Participants}

Forty adolescents aged 12 to 18 years old from a school in West London were recruited for the study (Table 1). The majority of the participants were of South Asian ethnicity $(n=35)$, while 3 were White Caucasian, 1 was African and 1 was of mixed race. None of the participants reported previous anxiety/mood or reading disorders, as ascertained through self-report. Participants under 16 years of age completed assent forms while their parents gave informed consent. Participants over 16 years gave informed consent. Ethical approval was received from the Central University Research Ethics Committee of the University of Oxford.

\section{Procedures and Materials}

An overview of experimental procedures is presented in Figure 1. In brief, participants first completed the Trait scale of the State-Trait Anxiety Inventory for Children (Spielberger, 1973) to assess anxiety symptoms. This was followed by an imagery exercise, in which participants were taught, with examples, to vividly imagine themselves in various scenarios with the aid of visual images (Lothmann et al, 2011). They were then instructed to apply this technique during the training phase in order to enhance the effects of CBM-I (Holmes et al., 2009). Participants were assigned to receive either positive $(n=20)$ or negative training $(n=20)$ using a random number generated sequence determined before testing. Immediately after training, participants completed a 10-minute picture-rating filler task. This was designed to neutralise any group differences in mood after training, which could confound group differences on the interpretation bias test and on anxious responses to the psychological challenge (mental arithmetic stressor performed under time constraints and to anticipated social evaluation). Participants rated anxiety levels using a self-reported Visual Analogue Scale (VAS) at 4 time-points across the study: Pre-training (VAS1), post-training (VAS2), pre-stressor (VAS3) and post-stressor (VAS4).

\section{CBM-I training task}

The training task consisted of sixty ambiguous situations that each ended with a word fragment (Figure 1). Participants were instructed to complete each fragment by typing in the first correct letter. Completion disambiguated the scenario in a negative or positive direction, dependent on training group. After the correct letter was selected, a comprehension question was presented, which 


\begin{tabular}{ll|c|cc} 
& & All Adolescents & Positive Training & Negative Training \\
\hline$n$ & & 40 & 20 & 20 \\
\% Female & 50 & 50 & 50 \\
Age & & $14.85(2.27)$ & $13.60(2.09)$ & $16.10(1.71)$ \\
\hline Trait anxiety score (STAI-C) & $40.28(7.01)$ & $38.75(7.85)$ & $41.80(5.86)$ \\
\hline Anxiety & VAS1 & $2.49(1.68)$ & $2.98(1.22)$ & $2.74(1.47)$ \\
(VAS scales) & VAS2 & $1.57(1.40)$ & $2.40(1.64)$ & $1.99(1.56)$ \\
\hline Word Completion RT & Block 1 & $2495.17(1450.71)$ & $1772.51(449.66)$ & $2133.84(1121.48)$ \\
(ms) & Block 2 & $1472.91(327.86)$ & $1657.41(357.80)$ & $1565.16(351.38)$ \\
& Block 3 & $1855.08(569.55)$ & $2058.35(877.40)$ & $1956.71(737.33)$ \\
& Block 4 & $1906.79(992.05)$ & $1610.23(435.26)$ & $1758.51(770.91)$ \\
& Block 5 & $1542.81(381.59)$ & $2124.53(1206.78)$ & $1833.67(931.23)$ \\
\hline Comprehension Questions & Block 1 & $86.50(12.68)$ & $83.00(12.18)$ & $84.75(12.40)$ \\
Accuracy (\%) & Block 2 & $95.50(5.10)$ & $92.50(5.50)$ & $94.00(5.45)$ \\
& Block 3 & $93.50(8.13)$ & $89.00(15.53)$ & $91.25(12.44)$ \\
& Block 4 & $91.00(16.19)$ & $95.00(6.07)$ & $93.00(12.24)$ \\
& Block 5 & $90.00(13.33)$ & $90.00(15.63)$ & $90.00(14.14)$ \\
\hline Comprehension Questions & Block 1 & $4530.94(803.10)$ & $4066.35(895.73)$ & $4298.64(872.03)$ \\
RT (ms) & Block 2 & $3530.31(656.27)$ & $3329.55(732.29)$ & $3429.93(693.83)$ \\
& Block 3 & $3731.46(749.70)$ & $3189.67(835.24)$ & $3460.56(830.03)$ \\
& Block 4 & $3507.43(751.44)$ & $3178.94(949.87)$ & $3343.18(861.58)$ \\
& Block 5 & $3351.57(881.31)$ & $3687.08(1280.29)$ & $3519.33(1083.50)$ \\
\hline
\end{tabular}

Table 1: Participant characteristics and task-related performance in positive and negative training conditions

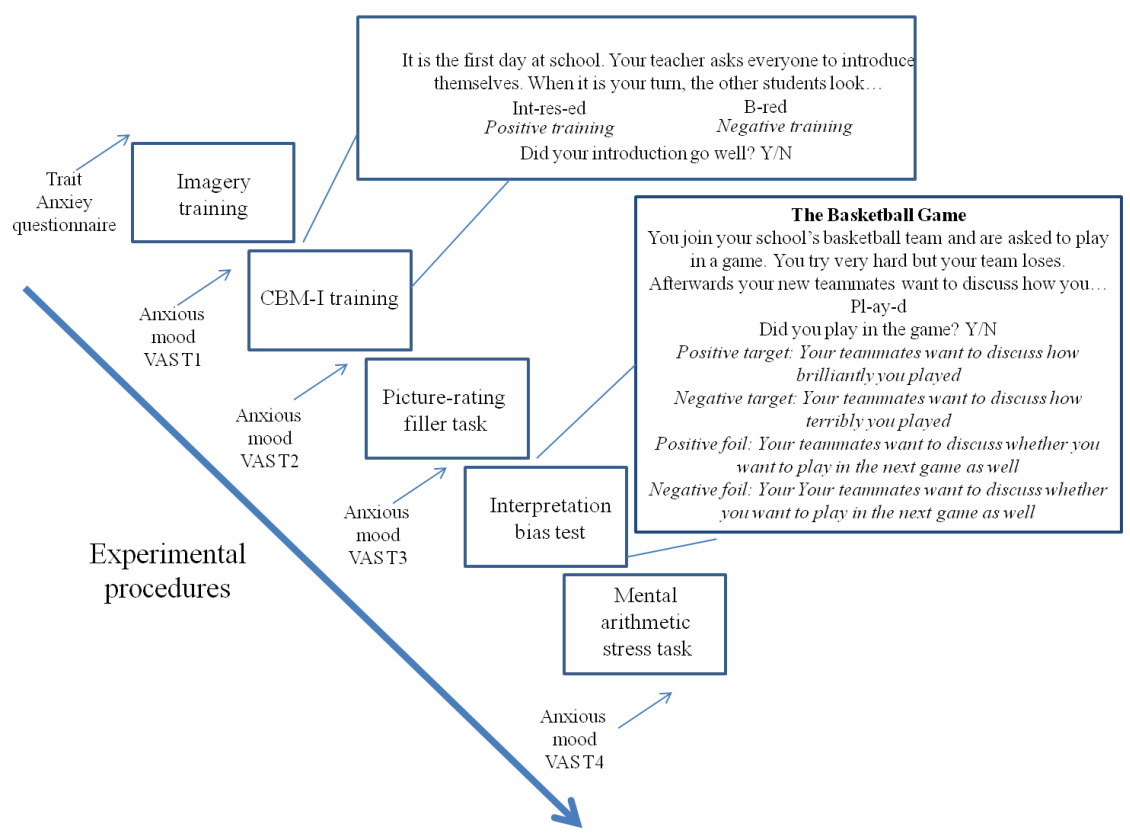

Figure 1: Summary of experimental procedures 
reinforced the valence of the interpretation, via immediate feedback about the response accuracy. The feedback consisted of a separate screen with 'Correct!' or 'Wrong!' appearing on-screen. To make these trials more relevant to adolescents, age-appropriate scenarios about friendship, romance, and school were used. In the current version of the task, each scenario was presented visually but accompanied with an audio voiceover to aid reading. High quality headphones allowed participants to listen to the various scenarios. In both positive and negative training conditions, scenarios were presented across 5 blocks. In each block, 1 scenario was resolved with the opposite valence (negative in the positive condition and positive in the negative condition) and 1 scenario was resolved neutrally, to mask the real purpose of the task.

\section{Picture-rating filler task}

This task consisted of 60 pictures of everyday objects, patterns and shapes which were presented for 10 second intervals on the laptop screen. Participants had to rate the pleasantness of each picture by either pressing the ' 1 ' key, for not pleasant and ' 9 ', for very pleasant.

\section{Interpretation bias test}

The test consisted of two phases (Figure 1). In the first phase, 10 ambiguously titled scenarios were presented visually with audio voiceover. Participants were given the same instructions as in the training phase. Unlike training items, completing word fragments did not disambiguate the emotional valence of the scenarios; the comprehension questions also had no emotional content. In the second phase, participants viewed the title of each scenario they had seen, followed by four statements relating to that scenario. Participants were informed that the sentences resembled the scenario, but none would use the same wording. They were instructed to rate statements on their similarity to the scenarios viewed in the first phase, on a scale from 1 to 4 (1: not similar at all; 2: not so similar; 3: similar; 4: very similar). These statements, ordered randomly, included one positive and one negative interpretation of the scenario (targets). The other 2 statements were also positively and negatively valenced but were not valid interpretations of the ambiguous scenarios (foils).

\section{Mental arithmetic stressor task}

Participants were asked to complete a timed mental arithmetic exercise verbally for 2 minutes. This exercise involved subtracting 7 from 555 on the first trial and subtracting 7 from the answer of the previous trial, on subsequent trials. Participants were also told that they would be video recorded during this task for a laboratory class at the University of Oxford. However in reality no participants were recorded.

\section{Visual analogue scales of anxious mood}

Participants indicated how much of each of 4 emotions they were feeling at that moment, on a line between "not (emotion) at all" $(0 \mathrm{~cm})$ and "very (emotion)" $(9.8 \mathrm{~cm})$. These emotions were taken from the original PANAS-C (Laurent et al., 1999) and included: nervous, worried, anxious, and scared. Scores were then averaged across these to create an anxious mood index across the four time-points.

\section{Statistical Analyses}

Wherever assumptions of normality were violated, log transformed data were used in the analysis. Group differences in training performance analysed reaction times to word fragments and the number of incorrect responses to comprehension questions using two mixed design ANOVAs. These contained training-condition (positive, negative) as the between-subjects factor and block (1-5) as the within-subjects factor. To examine the effectiveness of positive and negative training, a mixed design ANOVA with one between-subjects factor (training-condition: positive, negative) and two 
within-subjects factors (sentence-type: target, foil; and sentence-valence: positive, negative) was performed on similarity ratings from the Interpretation Bias Test. Significant interactions from this analysis were followed up by separate analysis of targets and foils, using two 2 (training-condition) $\times 2$ (sentence-valence) mixed ANOVAs. Finally, training effects on anxious mood were analysed separately for: (i) Changes from pre- to post-training using a $2 \times 2$ ANOVA with training-condition as the between-subjects factor and time $(1,2)$ as the within-subjects factor; and (ii) for changes from pre- to post-stressor again using the same $2 \times 2$ ANOVA but with data from time-points 3 and 4 (Figure 1). The Greenhouse-Geisser (G-G) correction was applied in these analyses. Where significant, age, gender and trait anxiety were included as covariates in these analyses.

\section{Results}

\section{Training Group Differences in Training Performance}

Reaction times (RT) to word fragment completion and RTs and errors to comprehension questions during training are presented in Table 1 for participants across training conditions. A significant main effect of time $\left(F(4,152)=4.19, p=.003, \eta_{p}^{2}=.10\right)$ emerged on RT data to word fragment completion. However this was modified by a significant training-condition-by-time interaction $\left(F(4,152)=5.89, p<.001, \eta_{p}^{2}=.13\right)$. While there were no training-condition differences at Blocks 2-4, at Block 1, the negatively-trained adolescents were quicker at completing word fragments than their positively-trained counterparts: $t(22.62)=2.13, p=.040$, Cohen's $d=0.89$. However, these patterns were reversed at block $5: t(38)=2.06, p=.047$, Cohen's $d=0.67$.

A significant main effect of time $\left(F(4,72)=3.05, p=.022, \eta_{p}^{2}=.14\right)$ also emerged on accuracy of responses to comprehension questions. This effect was driven by significantly lower $(t(39)=$ $-3.97, p<.001)$ accuracy scores during Block 1 relative to mean accuracy score across the entirety of the training phase. This effect was not modified by interactions with training group, nor did training group yield any main effect for comprehension question accuracy. A significant main effect of time $\left(F(4,72)=11.09, p<.001, \eta_{p}^{2}=.38\right)$ emerged on RT of responses to comprehension questions. Similar to the above effects, this was driven by significantly longer RTs $(t(39)=8.55, p<.001)$ to comprehension questions in Block 1relative to mean RTs across the entirety of the training phase.

\section{Training Group Differences in Interpretation Bias Test}

A significant 3-way interaction between training-condition, sentence-type and sentence-valence was found on similarity ratings $\left(F(1,38)=5.86, p=.020, \eta_{p}^{2}=.13\right)$, warranting separate analysis of training-condition and sentence-valence effects on targets and foils separately. For targets, a significant 2-way interaction between these variables emerged $\left(F(1,38)=61.23, p<.001, \eta_{p}^{2}=.62\right)$. As illustrated in Figure 2, compared to negatively-trained adolescents, positively-trained adolescents endorsed positive targets more often $(t(38)=5.44, p<.001, d=1.76)$ and negative targets less often $(t(38)=6.51, p<.001, d=2.11)$. Positively-trained adolescents also assigned higher similarity ratings to positive targets than negative targets $(t(19)=7.06, p<.001, d=3.24)$, whereas the opposite was true for negatively-trained adolescents $(t(19)=4.36, p<.001, d=2.00)$.

Similar findings characterised foil data (Figure 2), with a significant training-condition-by-foilvalence interaction $\left(F(1,38)=47.87, p<.001, \eta_{p}^{2}=0.56\right)$. Here, between-group differences also emerged where positively-trained adolescents assigned higher similarity ratings to positive foils $(t(38)=5.20, p<.001, d=1.69)$ and lower ratings for negative foils $(t(29.94)=3.29, p=.003, d=$ $1.20)$ relative to negatively-trained adolescents. Positive foils were endorsed more often by positivelytrained adolescents than were negative foils $(t(19)=9.88, p<.001, d=4.54)$.

\section{Training Group Differences in Anxious Mood Changes}

The first set of analysis examined training-condition effects on changes in anxious mood VASs from pre- to post-training (Table 1 ). Only a main effect of time emerged $\left(F(1,38)=11.74, p=.001, \eta_{p}^{2}=\right.$ $.24)$, in which anxiety scores decreased from pre-training to post-training. 


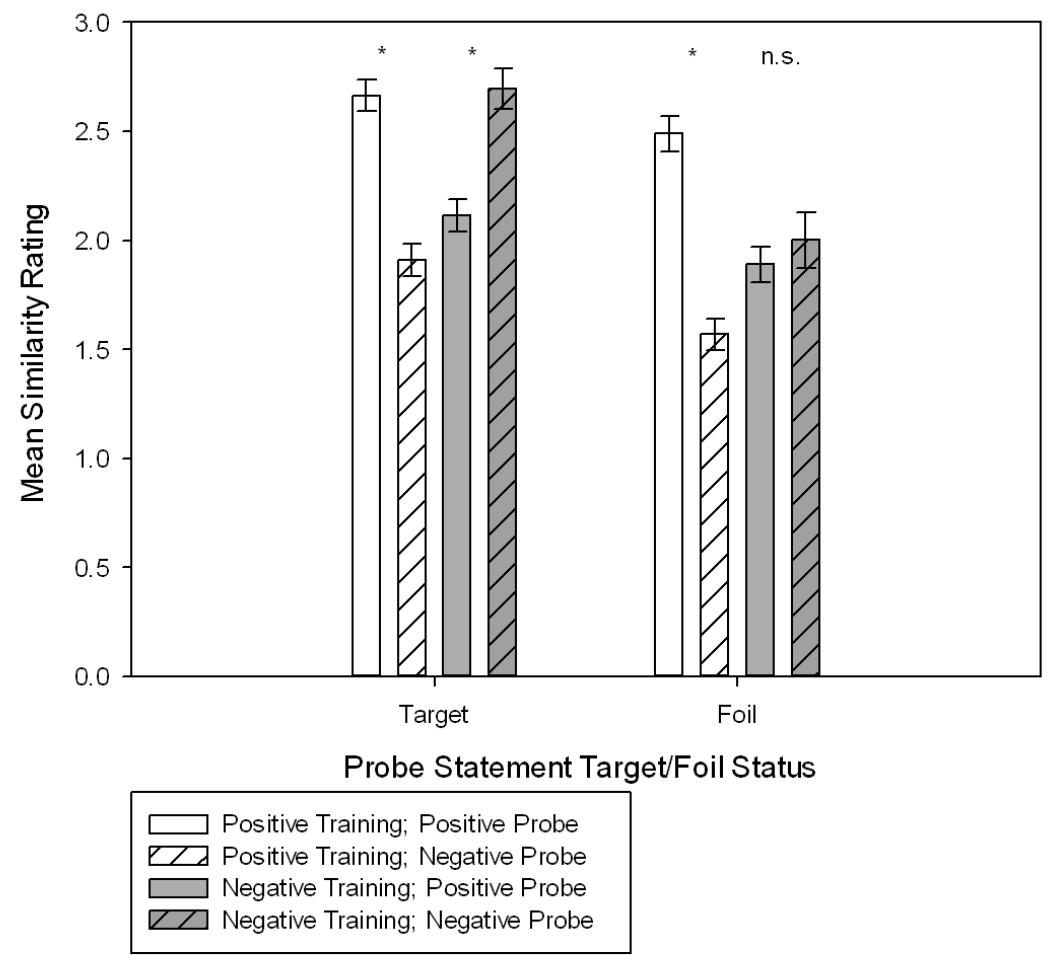

Figure 2: Similarity ratings of positive and negative targets and foils in each training condition 
The second set of analysis examined training-condition effects on changes in anxious mood VASs from pre- to post-stressor (Figure 3 ). A significant main effect of time $(F(1,38)=53.69, p<$ $\left.0.001, \eta_{p}^{2}=.59\right)$ suggested that for all participants, anxious mood ratings significantly increased from before to after exposure to the stressor task. However these effects were moderated by trainingcondition $\left(F(1,38)=4.34, p<0.044, \eta_{p}^{2}=.10\right)$. While no group differences on anxious mood were found prior to the stressor, after the stressor, positively-trained adolescents showed significantly lower anxious mood ratings.

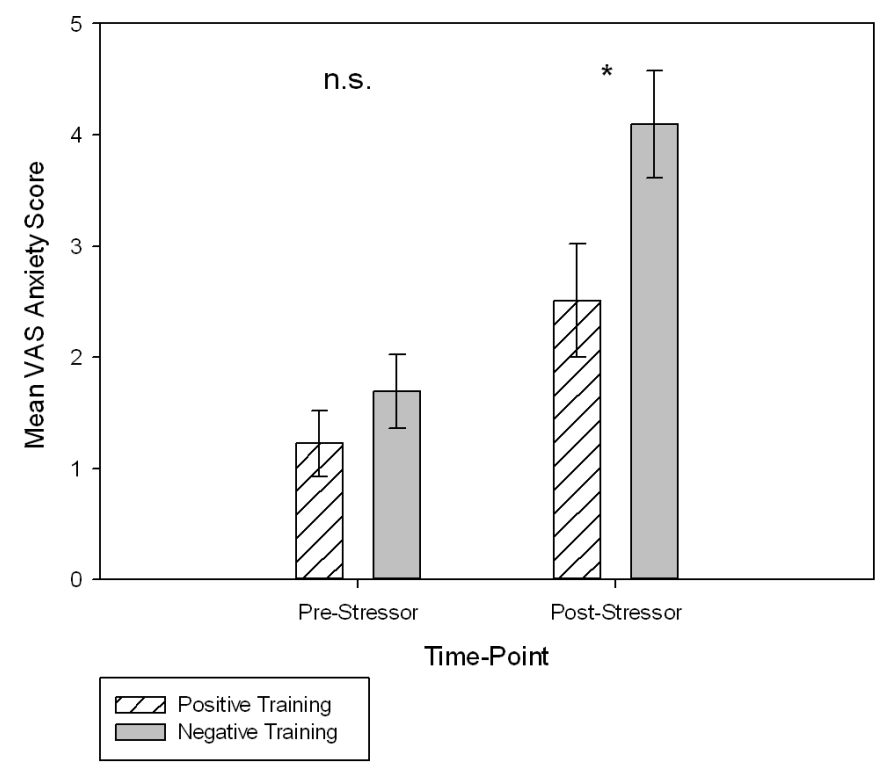

Figure 3: Anxiety ratings assayed using visual analogue scales before and after the psychological challenge

\section{Discussion}

The current data replicated our previous findings by showing that adolescent interpretational style is plastic, and moreover that positive interpretations can be generated through computerised training procedures. These data also suggest that the introduction of audio clips to aid reading does not appear to detract from the effectiveness of training. However the most exciting finding related to the attenuation of anxious responses to a psychological challenge in the positive relative to the negative training group. Tentatively, these findings support the view that 'boosting' positive cognitions in adolescents via CBM-I training may protect against the negative effects of stress.

Together these and other data (MacLeod et al., 2009) add to a growing optimism for the therapeutic potential of training tasks targeting and correcting negative cognitive biases, such as the tendency to draw negative interpretations and the tendency to allocate attention towards threatening stimuli. While there are some emerging adult (MacLeod et al., 2009) and child data (Muris et 
al., 2008; Muris et al., 2009; Vassilopoulos et al., 2009) supporting the efficacy of cognitive training tasks as a treatment adjunct for anxiety and mood disorders, it is clear from our data that these tasks are also capable of impacting emotional well-being across adolescents too, and in particular not just adolescents with diagnoses (Lau et al., 2010; Lothmann et al., 2011; Telman et al., 2011). This makes it an appealing tool for prevention particularly in adolescence, which has been linked to a heightened vulnerability for anxiety disorders. Further supporting this initiative are data showing that risk and resilience processes occurring during developmentally-sensitive periods can shape longer-term trajectories of emotional behaviour (Gross \& Hen, 2004). In other words, preventative interventions applied in adolescence may have the potential to attenuate adult outcomes. Finally, the accessible nature of these tasks through audio and visual channels could further add to their appeal, particularly among the current generation of adolescents who have grown up in an increasingly computer-literate world.

Despite these exciting implications, our results are subject to various limitations (though applying a positive interpretative style, one could argue that these limitations serve as guidance for future work!). First, we did not include a baseline measure of interpretational style. Thus there is the possibility that pre-existing differences in biased interpretation across training groups could confound training differences on the post-training interpretation bias test. Second, it is still unclear whether positive training effects on reducing anxious responses arise from genuine 'boosts' in positive interpretative style or from more general endorsement of positive material (i.e. a general positivity bias), given that positively-trained participants showed similar patterns of endorsement to foils as they did with targets. Additionally, it is unclear whether the relative attenuation of anxious reactivity effects in positive versus negative training groups was driven by increased anxiety-resilience as a result of positive training, increased anxiety-vulnerability as a result of negative training, or both. This issue could be addressed by comparing both positive and negative groups to a neutral or benign training condition. Third, although our stressor was effective, as indicated by the general increase in anxious mood from before to after the task, these results relied solely on self-reported measures of anxious responses, which are subject to demand characteristics. Future studies may consider supplementing self-reported data with physiological indices of anxious arousal, such as galvanic skin responses, heart rate measures or electromyography of the eye-blink startle response.

Finally only short-term effects of training on laboratory-based tasks were assessed in the present study. The degree to which training powerfully induces long-term changes in adolescence is not known. To be considered as a preventative tool in adolescence, a prerequisite will be to explore the stability of training-related improvements and the number and length of training sessions required to achieve this. A similar necessity applies to assessing the generalisability of training effects to real-world situations. Although our prior data suggest that training may shape interpretations of ambiguous material presented in the lab and retrospective perceptions of recent life events, further extensions of this work include investigation of training effects on responses to ambiguous situations and stressors as they occur in everyday life. In adolescence, such naturally-occurring emotional events are vast, evolving with changes in the immediate social (new and complex dynamics within peer groups) and educational environments (increasing importance of exams and changing curriculum) but also in the wider socio-cultural context, where there are increasing expectations of adolescents resuming adult-like responsibilities. Whether training can alter interpretations of these more complex ambiguous situations and emotional responses towards them needs to be examined carefully.

In summary, our data strongly suggest that CBM-I training tasks are capable of promoting positive interpretational styles and in attenuating anxiety during a psychological challenge in a sample of unselected adolescents. While aspects of this acquired positivity bias needs to be investigated further, these training tasks have the potential to lead to innovative, effective, and easy to deliver (e.g., internet-based) interventions to combat anxiety in young people. 


\section{Key points}

- Many adult anxiety disorders are rooted in adolescence, facilitating attempts to identify and implement prevention tools in this age range

- Prior data have shown that computerised cognitive training that modifies interpretation style can generate positive interpretations in adolescents

- The present study replicated these findings with a new audio-visual version of computerised training to aid adolescents less proficient at reading

- Our data further showed that these training-induced positive interpretations, attenuate adolescent anxious responses towards a mental arithmetic stressor performed under time constraints and anticipated social evaluation

- These data lay the groundwork for considering computerised cognitive bias modification of interpretations training task as a prevention tool for anxiety, across all adolescents rather than only those with diagnosed mental health issues

\section{Acknowledgements}

The authors would like to thank the students and teachers from Heston Community School who participated in this research. We would also like to thank Peter Ward for his help with software implementation. This study was funded by a small grant from the British Academy to JYL. SRB is funded by an ESRC studentship. 


\section{References}

Bar-Haim, Y. (2010) Attention bias modification (ABM): a novel treatment for anxiety disorders. $J$ Child Psychol Psychiatry, 51(8), 859-870.

Greenberg, P. E., Sisitsky, T., Kessler, R. C., Finkelstein, S. N., Berndt, E. R., Davidson, J. R., et al. (1999). The economic burden of anxiety disorders in the 1990s. J Clin Psychiatry, 60(7), 427-435.

Grey, S. J., \& Mathews, A. M. (2009). Cognitive bias modification - priming with an ambiguous homograph is necessary to detect an interpretation training effect. J Behav Ther Exp Psychiatry, 40(2), 338-343.

Gross, C., \& Hen, R. (2004). The developmental origins of anxiety. Nat Rev Neurosci, 5(7), $545-552$.

Hirsch, C. R., Hayes, S., \& Mathews, A. (2009). Looking at the bright side: accessing benign meanings reduces worry. Journal of Abnormal Psychology, 118(1), 44-54.

Holmes, E. A., Lang, T. J., \& Shah, D. M. (2009). Developing interpretation bias modification as a "cognitive vaccine" for depressed mood: imagining positive events makes you feel better than thinking about them verbally. Journal of Abnormal Psychology, 118(1), 76-88.

Lau, J. Y-F, Molyneaux, E., Telman, M. D., \& Belli, S. (2011) The Plasticity of Adolescent Cognitions: Data from a Novel Cognitive Bias Modification Training Task. Child Psychiatry and Human Development, 42(6), 679-693.

Laurent, J., Catanzaro, S. J., Joiner, T. E., Rudolph, K. D., Potter, K. I., Lambert, S., et al. (1999). A measure of positive and negative affect for children: scale development and preliminary validation. Psychological Assessment, 11, 326-338.

Lothmann, C., Holmes, E. A., Chan, S. W., \& Lau, J. Y. (2011). Cognitive bias modification training in adolescents: effects on interpretation biases and mood. J Child Psychol Psychiatry, 52(1), $24-32$.

MacLeod, C., Koster, E. H., \& Fox, E. (2009). Whither cognitive bias modification research? Commentary on the special section articles. J Abnorm Psychol, 118(1), 89-99.

Mathews, A., \& Mackintosh, B. (2000). Induced emotional interpretation bias and anxiety. $J$ Abnorm Psychol, 109(4), 602-615.

Mathews, A., Ridgeway, V., Cook, E., \& Yiend, J. (2007). Inducing a benign interpretational bias reduces trait anxiety. J Behav Ther Exp Psychiatry, 38(2), 225-236.

Mayer, R. E. (2008). Applying the science of learning: evidence-based principles for the design of multimedia instruction. Am Psychol, 63(8), 760-769.

Merikangas, K. R., Nakamura, E. F., \& Kessler, R. C. (2009). Epidemiology of mental disorders in children and adolescents. Dialogues Clin Neurosci, 11(1), 7-20.

Mezulis, A. H., Abramson, L. Y., Hyde, J. S., \& Hankin, B. L. (2004). Is there a universal positivity bias in attributions? A meta-analytic review of individual, developmental, and cultural differences in the self-serving attributional bias. Psychol Bull, 130(5), 711-747.

Muris, P., Huijding, J., Mayer, B., \& Hameetman, M. (2008). A space odyssey: experimental manipulation of threat perception and anxiety-related interpretation bias in children. Child Psychiatry 83 Human Development, 39(4), 469-480.

Muris, P., Huijding, J., Mayer, B., Remmerswaal, D., \& Vreden, S. (2009). Ground control to Major Tom: Experimental manipulation of anxiety-related interpretation bias by means of the "space odyssey" paradigm and effects on avoidance tendencies in children. Journal of Anxiety Disorders, 23(3), 333-340.

Murphy, R., Hirsch, C. R., Mathews, A., Smith, K., \& Clark, D. M. (2007). Facilitating a benign interpretation bias in a high socially anxious population. Behav Res Ther, 45(7), 1517-1529.

Pine, D. S., Cohen, P., Gurley, D., Brook, J., \& Ma, Y. (1998). The risk for early-adulthood anxiety and depressive disorders in adolescents with anxiety and depression disorders. Archives of General Psychiatry, 55, 56-64.

Sahakian, B. J., Malloch, G., \& Kennard, C. (2010). A UK strategy for mental health and wellbeing. Lancet, 375(9729), 1854-1855. 
Salemink, E., van den Hout, M., \& Kindt, M. (2007). Trained interpretive bias: validity and effects on anxiety. J Behav Ther Exp Psychiatry, 38(2), 212-224.

Spielberger, C. D. (1973). State-Trait Anxiety Inventory for Children. Palo Alto, Calif.: Consulting Psychologists Press.

Standage, H., Ashwin, C., \& Fox, E. (2009). Comparing visual and auditory presentation for the modification of interpretation bias. J Behav Ther Exp Psychiatry, 40(4), 558-570.

Stroud, L. R., Foster, E., Papandonatos, G. D., Handwerger, K., Granger, D. A., Kivlighan, K. T., et al. (2009). Stress response and the adolescent transition: performance versus peer rejection stressors. Dev Psychopathol, 21(1), 47-68.

Telman, M. D., Holmes, E. A., \& Lau, J. Y. (2011). Modifying adolescent interpretation biases through training: Effects on mood and stress appraisals. Submitted manuscript.

Van Ameringen, M., Mancini, C., \& Farvolden, P. (2003). The impact of anxiety disorders on educational achievement. J Anxiety Disord, 17(5), 561-571.

Vassilopoulos, S. P., Banerjee, R., \& Prantzalou, C. (2009). Experimental modification of interpretation bias in socially anxious children: Changes in interpretation, anticipated interpersonal anxiety, and social anxiety symptoms. Behav Res Ther, 47(12), 1085-1089.

Wilson, E. J., MacLeod, C., Mathews, A., \& Rutherford, E. M. (2006). The causal role of interpretive bias in anxiety reactivity. J Abnorm Psychol, 115(1), 103-111.

Wood, J. J. (2006). Effect of anxiety reduction on children's school performance and social adjustment. Dev Psychol, 42(2), 345-349.

Zimmermann, P., Wittchen, H. U., Hofler, M., Pfister, H., Kessler, R. C., \& Lieb, R. (2003). Primary anxiety disorders and the development of subsequent alcohol use disorders: a 4-year community study of adolescents and young adults. Psychol Med, 33(7), 1211-1222. 